\title{
Regarding the detectability and measurement of coronal mass ejections
}

\author{
Timothy A. Howard* \\ Southwest Research Institute, 1050 Walnut Street, Suite 300, Boulder, CO 80302, USA \\ ${ }^{*}$ Corresponding author: howard@boulder.swri.edu
}

Received 30 December 2014 / Accepted 19 June 2015

\begin{abstract}
In this review I discuss the problems associated with the detection and measurement of coronal mass ejections (CMEs). CMEs are important phenomena both scientifically, as they play a crucial role in the evolution of the solar corona, and technologically, as their impact with the Earth leads to severe space weather activity in the form of magnetic storms. I focus on the observation of CMEs using visible white light imagers (coronagraphs and heliospheric imagers), as they may be regarded as the binding agents between different datasets and different models that are used to reconstruct them. Our ability to accurately measure CMEs observed by these imagers is hampered by many factors, from instrumental to geometrical to physical. Following a brief review of the history of CME observation and measurement, I explore the impediments to our ability to measure them and describe possible means for which we may be able to mitigate those impediments. I conclude with a discussion of the claim that we have reached the limit of the information that we can extract from the current generation of white light imagers, and discuss possible ways forward regarding future instrument capabilities.
\end{abstract}

Key words. Sun - Corona - Coronal mass ejections - Space weather - White light imaging - Solar wind

\section{Introduction}

Coronal mass ejections (CMEs) are large eruptions of magnetic field and plasma from the Sun. They are of interest to the scientific community because they play an important role in the evolution of the solar corona and they are of interest to the technical community because they are the main drivers of severe space weather at the Earth. Their associations with solar evolution and space weather are well known and well documented (see, for example, the recent reviews by Webb \& Howard (2012) and Chen (2011) or the books by Howard (2011a, 2014)). Consequently our ability to detect and measure the properties of CMEs is a topic of great importance.

The arrival of the twin STEREO spacecraft (Russell 2008) enabled the measurement of CMEs in a manner that was not possible in prior years. This was primarily because STEREO were separated in azimuth from the Earth (and from each other) in the ecliptic plane, allowing simultaneous measurements of the Sun and CMEs from different viewpoints. The spacecraft also carry two heliospheric imagers that, when combined with the onboard coronagraphs, allow the continuous tracking of CMEs from their solar origins to $1 \mathrm{AU}$ and beyond. One promise of STEREO regarding CME measurement was their 3-D reconstruction and the identification of their location and trajectory in 3-D. The promise was that the ambiguous "sky plane" assumption that applies to coronagraphs would be removed using the multiple viewpoints, which would enable the accurate forecasting of arrival times and speeds at the Earth (see, e.g., Russell 2008). While progress has been made in techniques that attempt to reconstruct CMEs observed by the coronagraphs and heliospheric imagers on board STEREO (we review some of these in Sect. 5.1), this progress has been very slow and has not yielded the full promise of STEREO (the mission is now in its 9th year). The problem is not with the talents of the workers or the scientific validity of the methods they have developed, but rather it is the nature of the CMEs themselves and how we observe them that has hindered progress.

The STEREO mission has reached a critical stage, where the spacecraft are behind the Sun relative to the Earth and, at the time of writing, STEREO-B has been out of contact for several months and there are doubts as to whether it will be recovered. It is timely that we review the progress of STEREO toward CME detection and discuss CME observation in general. The purpose of this paper is to review the methods of CME detection, highlight the difficulties pertaining to CME measurement, and present the case that we may have reached the limit in the information we can extract about CMEs with contemporary instrumentation.

\section{Detection methods}

Coronal mass ejections are detected by a variety of methods. While the distinction is somewhat subjective, they can be divided into two categories: direct and indirect. Direct methods involve measurement of the properties of the CME itself, such as when they impact a spacecraft or via light scattered off the particles within them, while indirect methods involve processes that are caused by CMEs but are not intrinsically part of them. The former includes in-situ measurement, radio scintillation and white light observation (coronagraphs, heliospheric imagers), while the latter includes solar energetic particles (SEPs), radio bursts (Type II), and a variety of solar "surface" phenomena such as flares, filaments, coronal dimming, post-eruptive arcades, and coronal waves. Those indirect methods provide 
Table 1. Methods of measuring CME properties.

\begin{tabular}{ll}
\hline \hline Property & Method \\
\hline Frequency of occurrence & $\begin{array}{l}\text { Count the number of observed CMEs occurring over a given time period. } \\
\text { Distance (height) }\end{array}$ \\
$\begin{array}{c}\text { Select an easily identifiable feature near the CME leading edge (some choose a common position angle) and } \\
\text { measure its distance from the center of the Sun, calibrating to units of solar radii. } \\
\text { Measure the distances of the same feature in multiple images and plot the distance-time distribution. A linear } \\
\text { least-squares fit provides the speed; a second-order polynomial fit provides the acceleration. } \\
\text { Trace out the leading edge of the CME and calibrate to units of solar radii. }\end{array}$ \\
Geometry
\end{tabular}

a means to estimate CME location, such as information of associated active regions and filaments (see Webb \& Howard 2012 , for a review), and provide some information on the magnetic structure in the corona involving CMEs (e.g., Moore et al. 2001; Zhang \& Wang 2001; Fuller et al. 2008; Panasenco et al. 2011; Sun et al. 2012), but no reliable method for associating these observations with CME location has yet been established. Likewise a variety of models have been developed that describe the possible physics governing CME launch and evolution, which have had mixed success in reproducing observed CME properties (see, e.g., Chen 2011, for a recent review). While a great deal of progress has been made in improving our understanding of CMEs by way of solar disk measurements of associated phenomena, detailed in-situ studies, and modeling, for the purposes of the present review I confine my discussion to measurements made using white light instruments, as these enable direct comparison with other datasets, provide local and global measurements, and allow the continuous tracking across large distances. In other words, white light observations are the binding agents between the different kinds of observations and the models.

\subsection{A brief review of the history of CME observation}

The CME "discovery" paper is from the proceedings of the COSPAR meeting in 1972. Tousey (1973) described a cloud moving ahead of an erupting filament, observed with the first coronagraph to fly on a spacecraft (OSO-7). It later emerged that these "fast transient events" had been observed on the ground for decades (DeMastus et al. 1973). They were quickly associated with interplanetary shocks that had been measured by in-situ spacecraft since the early Space Age (Gosling et al. 1975), and identified by many as blast waves from solar flares (e.g., Dryer 1974). While the latter has now been debunked (see the paper by Gosling (1993) and the many papers surrounding the famous "solar flare myth" debate), the former is generally accepted to be a correct association. It emerged in the 1980s that highly structured magnetic features, called magnetic clouds, often followed interplanetary (IP) shocks (Burlaga et al. 1981; Klein \& Burlaga 1982) and it was accepted that (when present) they were the driver for the IP shocks observed in-situ (e.g., Klein \& Burlaga 1982; Lepping et al. 1990). By this time, the first generation of spacecraft coronagraphs (NRL's OSO-7 (Koomen et al. 1975) and HAO's instrument on Skylab (MacQueen et al. 1974)) had been replaced with the next generation; NRL's Solwind (Michels et al. 1980) and HAO's C/P on the SMM spacecraft (MacQueen et al. 1980). The latter enabled regular observations of a cavity trailing the bright leading front of CMEs observed by coronagraphs, that was ahead of the filament (Illing \& Hundhausen 1985). A picture emerged of a "classic" three-part CME configuration observed by coronagraphs (bright leading edge, cavity, filament), that was associated with a different three-part configuration observed in-situ (IP shock, sheath, magnetic cloud). It was suspected for some time that the cavity component of the three-part CME corresponded to the magnetic cloud in-situ (e.g., Forsyth et al. 2006) and this was recently confirmed observationally for at least one CME (Howard \& DeForest 2012a). Under this narrative, the shock and sheath in-situ components correspond to the bright leading edge in the coronagraph three-part CME, but the fate of the underlying filament component observed in coronagraphs remains open to debate. Little evidence exists of filament signatures at large distances from the Sun (Howard 2015b), and although periods of high-density cold plasma have been observed in-situ (e.g., Cane et al. 1986; Yao et al. 2010) it remains an open question as to whether these features are actually filaments and therefore how commonplace they are within CMEs at distances near $1 \mathrm{AU}$.

\subsection{White light CME measurement}

Throughout most of the history of white light CME observation, their measurement has been limited to the following quantities: frequency of occurrence, distance (height), speed (and acceleration), geometry, and mass. The methods for measuring these properties were established early (e.g., Gosling et al. 1974, 1975, 1976; Hildner et al. 1975; Howard et al. 1976; Poland et al. 1981), and by the early 1990s several statistical compilations had been produced (e.g., MacQueen 1980; Rust et al. 1980; Howard et al. 1985; Hundhausen et al. 1994). Hence, by the launch of the next generation of spacecraft coronagraphs in the mid-1990s (LASCO (Brueckner et al. 1995) on board SOHO, launched December 1995), the tools for basic CME measurement were well established and were used invariably by the vast majority of workers using LASCO data. For example, the two most widely used catalogs for CMEs, NASA's CDAW catalog (http://cdaw.gsfc.nasa.gov/CME_list/) and the NRL catalog (http://lasco-www.nrl.navy.mil/index. $\mathrm{php}$ ? $\mathrm{p}=$ content/cmelist), provide these same measurements for CMEs observed by LASCO. Table 1 provides a list summarizing the methods used for CME measurement. Determining the mass of a CME is somewhat more involved; I discuss that in Section 2.4.

While the methods shown in Table 1 are very straightforward, inherent within them are a number of assumptions that have been applied to simplify the analysis of coronagraph images. Some of these are appropriate, as applying the full treatment needed to accommodate all factors provides an insignificant difference to the measured result, while others can potentially change the measured result a great deal. Others still break down when we move away from the near-Sun environment observed by coronagraphs, such as the regions observed by heliospheric imagers. 
T.A. Howard: The detection and measurement of coronal mass ejections
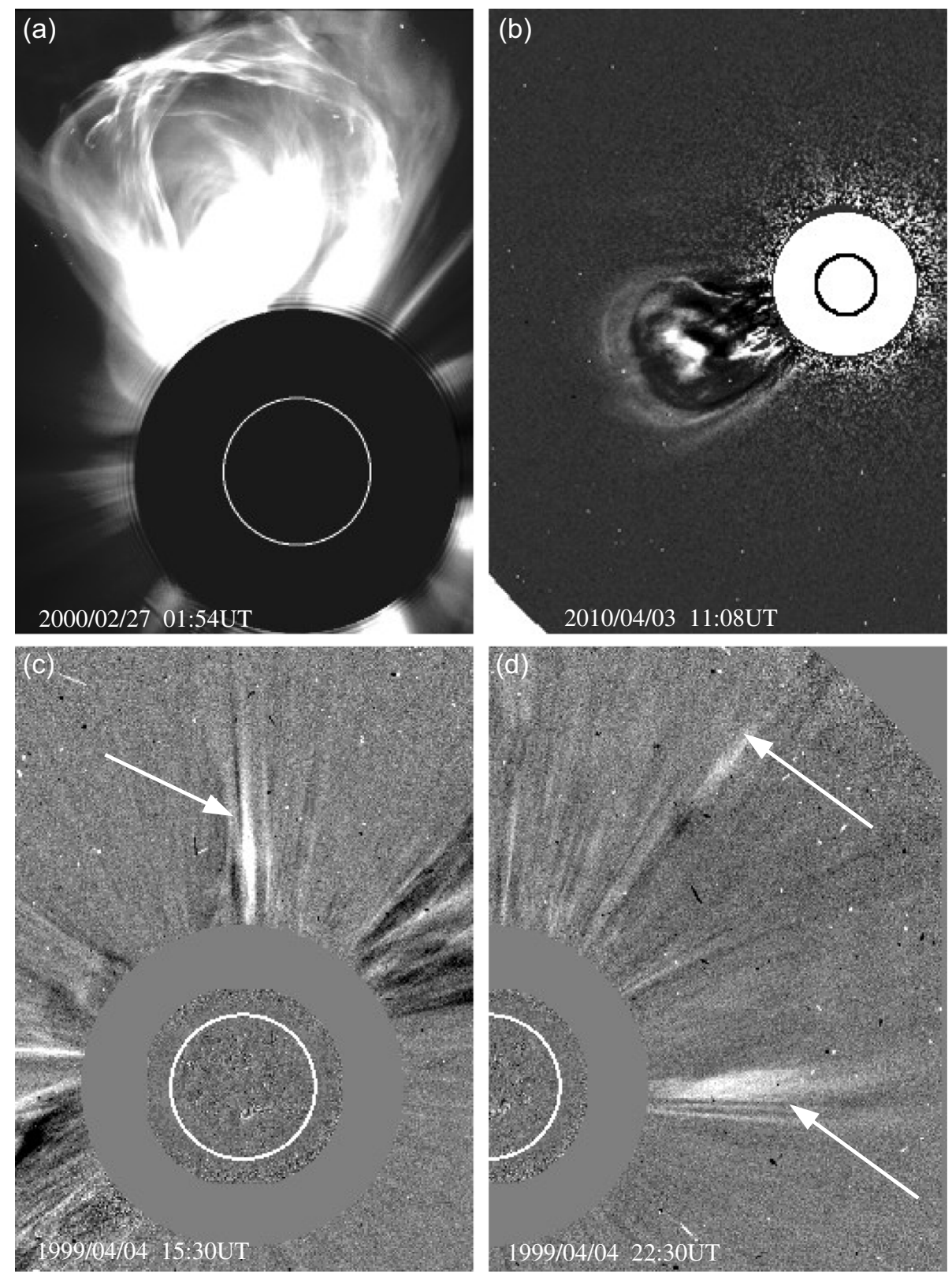

Fig. 1. Images of CMEs with white light coronagraphs, highlighting the problems associated with CME definition. The top row shows two images of CMEs from (a) LASCO/C2 (standard background subtracted) and (b) STEREO-A/COR2 (running-difference); these are universally accepted as CMEs by the scientific community. The bottom row shows LASCO/C2 running difference images of narrow ejecta over which the community is divided as to whether to identify them as CMEs. Both images are from the same day (4 April 1999), but the one shown in panel (c) is identified as a CME in the widely used CDAW catalog, while the two shown in panel (d) are not. The author speculates that, in this case, the decision for CME identification at the discretion of the observer.

\subsection{Autonomous detection}

The vast majority of CME measurements have been performed manually. However, the last decade has seen the emergence of autonomous CME detection techniques. Along with the obvious benefits of speed and the removal of the need for a user, autonomous detection has the additional advantage that the same selection criteria are applied across the entire dataset. This effectively removes the ambiguous detection arising from observer bias, such as those highlighted in Figure 1. The disadvantage is that the selection criteria are entirely reliant on the parameters of the autonomous method, providing problems with the balance of false positive identification and event selection. Further, autonomous detection cannot distinguish between properties such as multiple eruptions or CME substructure that are explored in Section 5.2. Several methods of autonomous detection of CMEs have been developed, among them are CACTus (Robbrecht \& Berghmans 2004), ARTEMIS (Boursier et al. 2009b), and CORIMP (Byrne et al. 2012) for coronagraphs, and AICMED for heliospheric imagers (Tappin et al. 2012).

\subsection{CME mass}

The mass of a CME is determined by applying the physics by which the light from the electrons within the CME reaches the imager. The theory of Thomson scattering applied to the Sun dates back to the 19th century (Schuster 1879), but the modern version of this theory was developed for the solar corona by Minnaert (1930) and Billings (1966). The standard method of 
calculating CME mass relies on the relationship between the scattered radiance (surface brightness) of a volume of electrons and the volume density, which is dependent upon only two linearly independent variables: the scattering angle $\chi$ (the angle between the solar radius vector and the observer, through the scattering point) and the angular width $2 \Omega$ of the Sun relative to the scattering point. Historically, workers have relied on the theory presented by Billings (1966) to perform the mass calculation but it was later discovered that confusion between radiance and intensity in this text has led to incorrect formulation in the theory. Consequently, the author of the present review and colleagues re-worked the theory along the lines of Minnaert (1930), providing a modern version to accommodate both for the oversight by Billings and for the analysis of white light images at large angles from the Sun (see Howard \& Tappin 2009; Howard \& DeForest 2012b, for the reworking of this theory).

Using this theory, the method for determining the mass of a CME is straightforward:

1. Measure the area on the image occupied by the CME by either drawing a polygon around the CME or measuring the CME on a pixel-by-pixel basis;

2. Convert each pixel, which is calibrated in units of radiance, into mass, using the theory of Thomson scattering. Note that this calculation requires a value for $\chi$, which requires a direction or 3-D location of the CME or pixel. It also requires a conversion from radiance to intensity, as it is the latter that is directly proportional to the mass;

3. Perform the integral across the whole CME, i.e., add together the pixel-mass values to determine the total mass of the CME.

It is important to note that the final step in this procedure (the integral across the $\mathrm{CME}$ ) is crucial when using the traditional method of CME mass calculation, as this method relies on the theory of Billings (1966), which contains the aforementioned error regarding radiance and intensity. This error only applies when the calculations are made at the small (i.e., single electron) level, but cancels when the integral is applied across the entire volume of the CME (Howard \& Tappin 2009).

The application of this method is dependent upon three important assumptions:

1. That the white light images are correctly calibrated to units of radiance. Commonly each pixel in the image is reported in "solar brightness units" $\left(B_{\odot}\right)$, where $1 B_{\odot}=$ $2.3 \times 10^{7} \mathrm{Wm}^{-2} \mathrm{SR}^{-1}$;

2. That all of the observed radiance arises from Thomson scattered light and not, say, from emission from spectral lines in the visible light spectrum;

3. That the material scattering the observed light is entirely enclosed within the CME.

As I explore in this paper, none of these assumptions are especially safe. Nonetheless, as is the general consensus of the white light imaging community, one must start somewhere.

\section{Unresolved questions regarding CME measurement}

It might surprise the unacquainted reader, but a number of seemingly basic questions remain unresolved with regard to CMEs. There is not yet, for example, a universally accepted definition for what a CME is or what signatures are telltale signs of CMEs in white light and what are not. This ambiguity results in different workers yielding different measurements of the same $\mathrm{CME}$, creating confusion and uncertainty. In this section I describe two important unresolved questions and explore the obstacles towards their resolution.

\subsection{What is a CME?}

Forty years after the discovery of CMEs by white light coronagraphs, a discussion session was held at the 2013 Solar, Heliospheric, and INterplanetary Environment (SHINE) Workshop in Buford, Georgia. Its title was "What Exactly is a Coronal Mass Ejection?" The session was concluded without resolution.

There are degrees at which the identification of CMEs is made with varying levels of confidence. On one end, we have those that are identified unambiguously by all; these include classic three-part CMEs and large-scale bubble-shaped eruptions such as those shown in Figures 1a and 1b. Likewise when observing in-situ, features that exhibit the three signatures defined by Burlaga et al. (1981) - increased $B$ field, decreased temperature, and a smooth rotation of the $B$ field vector - are universally recognized as magnetic clouds. The problem arises when we consider smaller eruptions that have a geometry that is less well defined in the white light datasets. For example, consider the features shown in Figures 1c and 1d. The feature indicated in panel (c) is identified as a CME in the NASA CDAW catalog but the two indicated in panel (d) are not, despite their similarity in appearance. Since all three of these occurred on the same day, it seems that the definition of the $\mathrm{CME}$ is at the discretion of the observer.

Ideally, one would prefer to define a CME in physical terms, and the most obvious path here is to define it in terms of the presence of a magnetic flux rope at its core, since it is accepted that this component contains the strongest magnetic field in the CME structure and therefore governs its formation and evolution, at least when the CME is in the corona (see Sect. 4.4). The problem is that we do not always see flux ropes even when one may be present. When observing with coronagraphs, we use cavities as the most forthcoming evidence of the presence of a magnetic flux rope; the cavity is a signature of density depletion caused by pressure balance effects and increased magnetic pressure, decreased thermal pressure, and resulting expansion that is known to occur within flux ropes (e.g., Low 1993). Consider Figure 2, which shows two images of the same CME observed from different angles. When observed from one direction (in this case, STEREO-A), we see a cavity, but when observed from a different direction (STEREO-B, $83^{\circ}$ away) the cavity cannot be seen. Had we been only able to observe this CME using STEREO-B, we would not be able to conclude that it contained a flux rope. Clearly we cannot use this criterion to define a CME observed in white light; we are bound by our observing capabilities.

Let us return to the small features shown in Figures 1c and $1 \mathrm{~d}$. It seems unlikely that they contain magnetic flux ropes, certainly there is no evidence of cavities within them, but should we call them CMEs? In the strictest sense, they are ejections of mass in the corona and they therefore fit the highly general term "coronal mass ejection". However, mass is constantly being ejected through the corona since its natural state is one of expansion. At what point does a mass ejection become part of the general fabric of the solar wind, and does this change if we use a detector with a different sensitivity? These are the 
T.A. Howard: The detection and measurement of coronal mass ejections
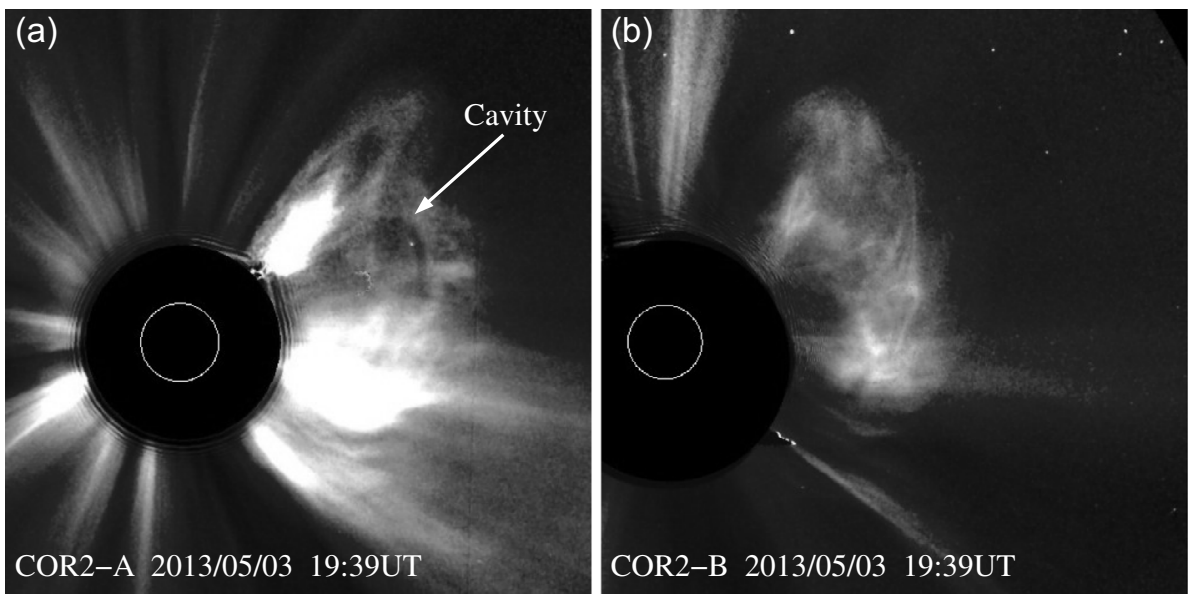

Fig. 2. Sometimes the cavity component of a CME is not easily detectable and may depend on the viewpoint. Here we see two images of the same CME taken from the same time from observers at different locations. (a) STEREO-A/COR2 image from 2013/05/05 19:39UT; (b) STEREO-B/COR2 image taken at the same time. A cavity is visible in COR2-A (indicated with the arrow), but not in COR2-B. At this time the STEREO spacecraft were separated by a (heliographic) longitudinal angle of $83^{\circ}$

questions that remain unresolved, and while I explore them no further in this review I present them as examples of the problems associated with CME measurement. Henceforth, when I use the term "CME" I refer to those features that are unambiguously recognized as such by the community, such as those shown in Figures 1a, 1b, and 2. As we discover throughout this review, measuring just that class of CMEs is sufficiently difficult to warrant this focused exposition.

\subsubsection{The bright background}

Before we move from the definition of a CME, the issue of background and its affect on CME identification needs to be addressed. Coronagraphs and heliospheric imagers are required to operate with very high sensitivity in order to observe CMEs, the brightest of which have radiances of the order of $10^{-10} B$. in the field of view of coronagraphs, and $10^{-13} B_{\odot}$ in heliospheric imagers. Consequently, the images are sensitive to the much brighter $\mathrm{K}$ and $\mathrm{F}$ corona, and to background planets and stars including the Milky Way. The nature of this background is very well explored in the literature and I will not revisit it here. The reader can refer to Thompson et al. (2010) and Frazin et al. (2012) for recent papers involving background subtraction for white light coronagraphs, and DeForest et al. (2011), Tappin et al. (2012), and Howard et al. (2013b) for those involving heliospheric imagers.

The reader is reminded that one must be very careful not to remove part of the signal one wishes to measure; for example, crude methods such as running-difference subtraction are of no value for measurements requiring the CME radiance, as part of the $\mathrm{CME}$ radiance is invariably removed when the prior image is subtracted. Likewise should one choose a base-difference image one must be careful that features removed from the selected image do not later become part of the CME mass that need to be accounted for.

\subsection{How does one determine what to measure?}

Consider the CME shown in Figure 3a. It is a classic three-part CME with a well-defined leading edge, cavity and underlying filament, observed by the LASCO-C3 coronagraph on board
SOHO. At first glance, measuring the properties of this CME would appear to be straightforward; we simply follow the methods outlined in Table 1. How do we decide what feature in this CME to measure? Would it matter if we measured, say, the feature labeled as "(i)" in the figure, rather than that labeled "(ii)"? If it is the leading edge that is sought, perhaps we should measure the edge of the diffuse material ahead of the brightest part of the feature ahead of the cavity, labeled "(vii)". Now that our vision has moved away from the overlying CME picture we see many substructures within the CME; I have labeled nine of them in Figure 3a. Are these part of the same CME, or are they different structures? To illustrate the importance of these questions, consider the plots shown in Figure $3 \mathrm{~b}$, which show the height-time distributions for each of the nine indicated features using measurements only from the $\mathrm{C} 3$ data. These plots yield a large variance $(\sigma=73$ or $20 \%$ of the mean) of speeds, ranging from $245 \mathrm{~km} / \mathrm{s}$ to $450 \mathrm{~km} / \mathrm{s}$. Had we been using these speed measurements to predict the time of arrival of this CME at $1 \mathrm{AU}$, our predictions for the transit time would have varied from 3.8 to 7.1 days.

Now consider the CME shown in Figure $3 \mathrm{c}$; this one observed by the LASCO-C2 coronagraph with an EUV image of the Sun included within. This is known as a halo CME, following the definition of Howard et al. (1982), and shows a CME that has a large component directed along the Sunobserver line such that its geometry encircles the Sun. It has been established that halo CMEs are strong indicators of CMEs heading toward the Earth (Howard et al. 1982, 1985) and are therefore vital signatures for space weather forecasting (e.g., Crooker 2000). Keeping with the theme established in the last paragraph, I have highlighted 20 features in this single image. Some appear to be enclosed structures rather than part of the enveloping CME structure. Again the question arises: Which feature do we measure? What impact would the measurements of different features have on the speed determinations, and therefore the prediction of the arrival time of this $\mathrm{CME}$ at Earth? It is noteworthy that this particular CME was one of the famous "Halloween storm" events, which did impact the Earth and caused numerous deleterious affects to our technological infrastructure when it arrived (e.g., Bao et al. 2005; Farrugia et al. 2005; Gopalswamy et al. 2005; Malandraki et al. 2005; Manchester et al. 2008). 

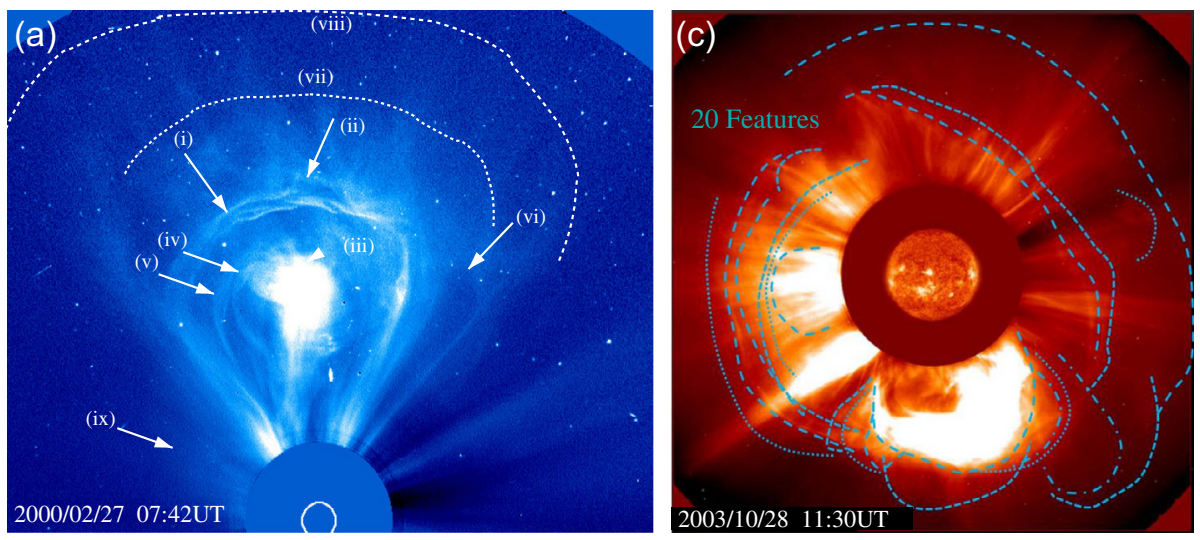

(b)

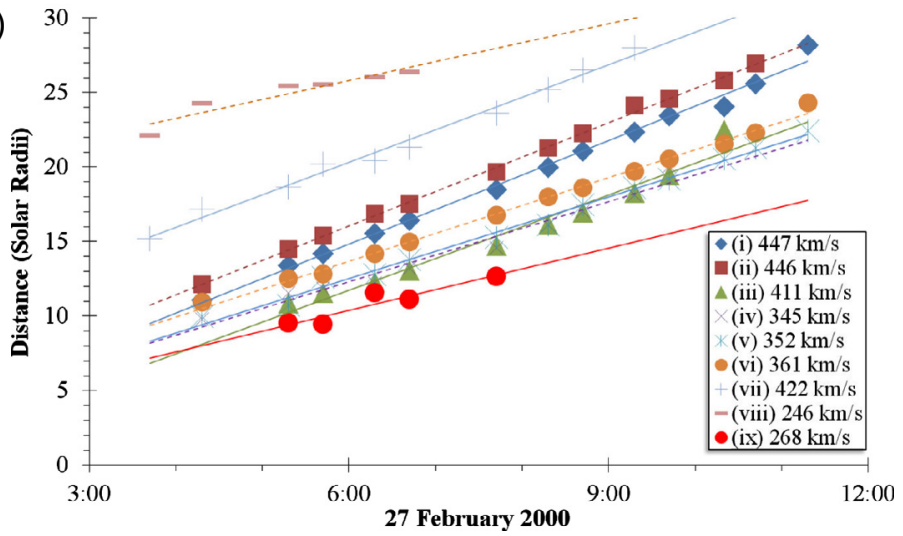

Fig. 3. Selecting features in coronagraph images can be troublesome and provide widely different results. (a) A LASCO/C3 image of a wellknown CME observed on 27 February 2000 (image provided courtesy of NASA). Nine distinct features have been identified in the image and are labeled (i)-(ix), (b) the height-time plots for each of the nine features, and the speed of each determined from the least-squares linear fit applied through each, (c) a LASCO/C2 image of one of the well-known "Halloween" CMEs (image also available courtesy of NASA), with 20 distinct features selected.

\section{Problems with CME measurement}

The questions raised in Section 3 address many of the practical issues associated with CME measurement in white light images, but these are contingent on the physical restraints imposed by the CMEs themselves, the environment through which they travel and the means by which our imagers detect them. These impose limits upon our ability to extract information from the images to a level of accuracy beyond which we cannot achieve because the information is simply not available. In this section I describe the restraints imposed by the physics of CMEs and how they are detected.

\subsection{The Thomson scattering assumption}

The analysis described in Section 2.2 depends on the assumption that all of the light observed by white light imagers has been Thomson scattered. This is especially true of mass calculation, since the conversion from radiance to mass entirely depends on this assumption. The question arises as to whether this is a safe assumption to make. We know, for example, that low in the solar atmosphere much of the brightness from the filament component is due to $\mathrm{H} \alpha$ emission, which is in the visible light range and therefore detectable by the broadband white light imagers we use in coronagraphs. We also know that the filament is often the brightest part of the CME in coronagraph images, and my cursory measurement of the CME displayed in Figure 3 a reveals that filament contributing around
$40 \%$ of the total $\mathrm{CME}$ intensity in the field of view of LASCO/C3 (i.e., at distances larger than 4 solar radii from the Sun). Given that the vast majority of CME mass measurement techniques include the filament with their measurements of the CME, it is important to determine what contribution of the observed intensity is due to Thomson scattering, and what is due to other kinds of emission.

The topic of $\mathrm{H} \alpha$ inclusion in white light coronagraphs involved much discussion in the early days of CME observation (V. Pizzo, private communication, 2014), and has been explored in a number of early publications (e.g., Poland \& Munro 1976; Schmahl \& Hildner 1977; Athay \& Illing 1986; Illing \& Athay 1986). It appears that the consensus was reached that line emission was sufficiently diminished by the time the filament reached distances of a couple of solar radii from the Sun, to be insignificant compared with the Thomson scattered contribution. However, recent work by Mierla et al. (2011) demonstrates that $\mathrm{H} \alpha$ emission is likely present in the STEREO/COR1 field of view. They found a significantly low polarized signal in the bright filament component of a CME and determined its location to be very close to the plane of the sky relative to the observer. Since Thomson scattered light polarization is maximized at this location (see Sect. 4.2) they concluded that the light was due to $\mathrm{H} \alpha$ emission, as photospheric $\mathrm{H} \alpha$ is known to have an unusually low polarization due to $\operatorname{Ly} \beta$ absorption (Poland \& Munro 1976). The author has recently published two papers that explore a filament measured at large distances from the Sun (Howard 2015a, 2015b). 
T.A. Howard: The detection and measurement of coronal mass ejections

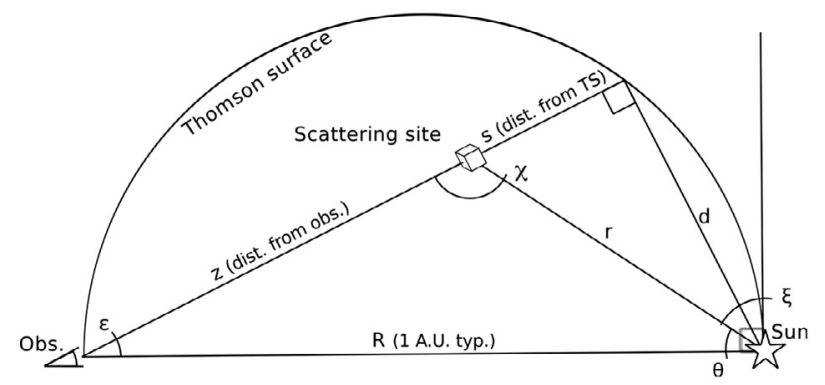

Fig. 4. Diagram showing the scattering of light from a volume of electrons (from Howard et al. 2013a). The location of the scattering volume (site) is shown, along with the angles used in the present review.

\subsection{The nature of Thomson scattering}

The theory of Thomson scattering is well established and I will not revisit it here (see Howard \& Tappin 2009; Howard \& DeForest 2012 b, for recent developments in this theory regarding white light imaging). I mentioned in Section 2.4 that the scattered radiance from a volume of electrons is dependent on the the scattering angle, $\chi$, and the angular width of the Sun, $2 \Omega$, relative to the scattering volume. The geometry is shown in Figure 4 at large distances, where the Sun tends toward a point source (i.e., $\Omega$ is small). Plotting the scattered radiance as a function of the angle from the plane of the sky relative to the observer, $\xi$, yields the distributions shown in Figure 5. I show these for a selection of lines of sight, each corresponding to an elongation angle, $\varepsilon$, from the Sun. Figure 5a shows the unpolarized scattered radiance $d B$, with the angle from the sky plane where the line of sight reaches the closest approach to the Sun indicated with a vertical bar. The physics of Thomson scattering requires the scattered radiance to maximize here, but note that the distribution remains flat at this maximum value for a large range of $\xi$. Howard \& DeForest (2012b) termed this the "Thomson plateau".

The important physical consequence is that a scattering volume can be located across a very large distance along a LOS (some $50^{\circ}$ from the point of closest approach) before any significant change in $d B$ is observed. This effectively annihilates any 3-D information from the white light images themselves, when observing total radiance.

Figure $5 \mathrm{~b}$ shows the same plot as in panel a, but for the scattered polarized radiance $d(p B)$. The Thomson plateau is absent here, enabling the possibility for retaining 3-D information from the white light images. This is discussed further in Section 5.1.

\subsection{CMEs are optically thin}

It has been known for centuries that the corona is optically thin. In ancient terms, this meant that comets could pass behind the corona and still be observed even though we did not know until the end of the 19th century that the corona had a solar origin (see, e.g., Billings 1966, and references therein). In modern terms, this means that the particles comprising the plasma within the corona do not scatter light from their neighbors, resulting in it being diffuse at most wavelengths. This likewise corresponds to transients within the corona, such as CMEs. Optical thinness has the advantage that, assuming that all of the observed light is Thomson scattered (Sect. 4.1), it validates the mass calculation tools (Sect. 2.4), since the calculated mass will be largely invariant across large distances along the LOS. The disadvantage is that it reduces our ability to distinguish between plasma that is part of the CME and plasma that is elsewhere along the LOS. The measured radiance contains no information as to where along a LOS the scattering volume of electrons lies or the level to which multiple volumes contribute to the measured radiance.

\subsection{CMEs are 3-D objects}

When observing a CME as it appears in a white light image, it is tempting to regard it as a two-dimensional plane or as a spherically symmetric feature. This may be suitable (and even appropriate) when considering small CMEs but not for larger ones.

Recall from Section 2.1 that many CMEs observed in-situ exhibit signatures known as magnetic clouds, and a white light cavity component, believed to be the magnetic cloud, that is approximately circular in shape. It is widely believed that these are manifestations of a magnetic flux rope (e.g., Webb 1988; Sturrock 1989; Crooker et al. 1990; Bothmer \& Schwenn 1998) that is responsible for the physics governing its behavior and appearance (Sect. 3.1). The most basic form of flux rope is of a coil, perhaps resembling a spring in structure. While it is assumed that the magnetic infrastructure comprising a CME is substantially more complex than this, I use the basic example to illustrate a point about geometry and perspective.

Consider the three scenarios depicted in Figure 6. In the first case (panel a), we have an ideal coil linearly aligned along the plane of the observer; in the case depicted in panel (b) it moves with one end leading; and in panel (c) we have the center leading the flanks. I show each scenario viewed from three coplanar, but separate locations. Given that CMEs are optically thin (Sect. 4.3) and the nature of their scattering allows us to see along the LOS with equal radiance (Sect. 4.2), a white light imager would display this entire structure projected into the sky plane. We can see how vastly different 2-D configurations emerge as we vary the scenario and viewing location.

In a separate color, I have also highlighted a fixed feature on the ideal coil, which remains in the same place at all times and in all three locations. I have selected a location at the bottom of the third coil. Note the following:

1. Unless observing the coil edge-on, it is difficult to identify where in the 3-D structure of the spring the feature lies;

2. The location of the feature relative to the observed "CME" loop structure changes with scenario and observing location.

Under most conditions we observe this ideal coil as a series of loops at different locations, but generally part of an overall collective. This might be interpreted as multiple eruptions or multiple magnetic structures, but in this case they are part of the same ideal coil. Figure 1a shows a strikingly similar comparison between one of the scenarios depicted in Figure 6 and a CME observed by a white light image.

We can continue to expand upon the level of complexity surrounding the scenarios. It is physically reasonable to allow for the coil loops to expand as they erupt and even for them to expand at different rates. Different segments can move at different rates or even different directions (Howard \& DeForest 2014). 

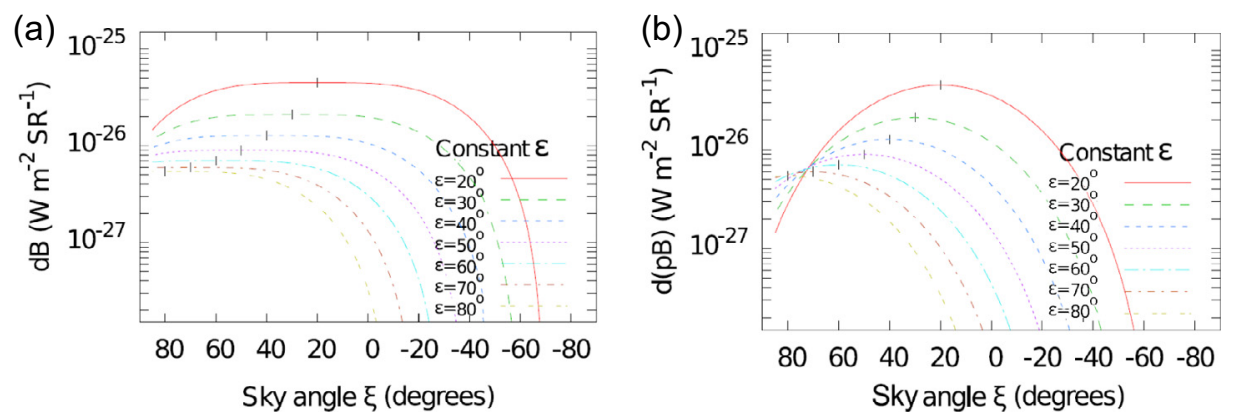

Fig. 5. Plots of scattered radiance as a function of angle from the sky plane, $\xi$, relative to the observer, shown across a range of elongation angles (lines of sight). The vertical bars represent the angle of closest approach to the Sun of each line of sight. The ordinate shows (a) the total scattered radiance $(d B)$ and (b) the (excess) polarized component of the scattered radiance $(d(p B))$. From Figure 2 of Howard et al. (2013a).

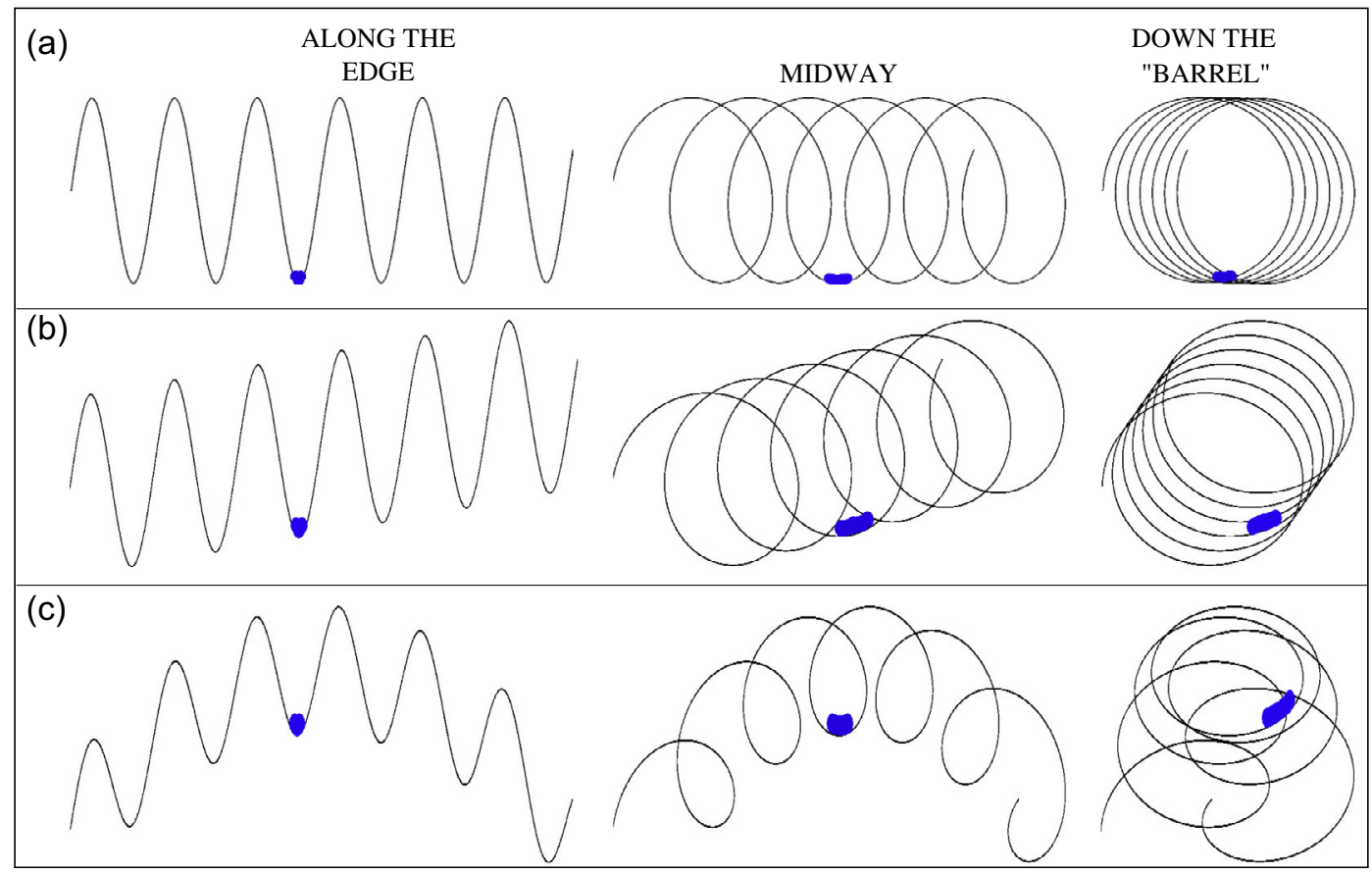

Fig. 6. Illustrations of the effects of geometry and perspective when measuring CMEs. The simple case of a symmetric circular coil, intended to represent an oversimplified flux rope, is presented in three scenarios: (a) completely flat; (b) at an incline, such as if it erupted with one end leading the other; and (c) with the center leading the flanks of the coil. In the case of white light imagery, a fictional observer would observe these configurations in their entirety, and a section at the bottom of the third coil has been highlighted in blue to demonstrate the variation of its location in each scenario. Note that the alignment referred to as "Down the Barrel" is slightly off-axis to highlight the location of the blue feature in the overall structure.

Flux ropes can be twisted or kinked (e.g., Fan \& Gibson 2003, 2004; Török \& Kliem 2003, 2005), they may interact with the surrounding coronal field in different ways (e.g., Antiochos et al. 1999; Lynch et al. 2008), or the flux rope may be subject to a variety of onset scenarios (e.g., Martin et al. 1985; Sturrock 1989; Zhang \& Low 2004; Rachmeler et al. 2009). Howard \& DeForest (2014) explored a case where a single flux rope underwent three separate onset mechanisms before it was completely disconnected from the Sun. I discuss this study further in Section 5.2.

\subsection{Calibration issues}

Commonly used white light imagers are calibrated into units of solar brightness - I mentioned this in Section 2.4. The accuracy of such calibration is crucial for mass measurements and likely affects other measurements such as CME location as well. Certainly, the contrast of a given image of a CME will affect where one decides upon the location of the leading edge, but this is more of a display issue than a calibration issue. It has been assumed that the white light imagers have been adequately calibrated (see, e.g., Morrill et al. 2006; Bewsher et al. 2010), but in the interests of completion I will briefly explore a possible problem here.

Consider the plots shown in Figure 7, which follow the technique devised by C. de Koning (C. de Koning, private communication, 2014). They represent the mass of a particular CME calculated using the standard technique described in Section 2.4. Rather than assigning a particular value of the angle $\xi$ from the plane of the sky, I plot the masses determined from a range of $\xi$ values, and show these plots for three coronagraphs observing the same CME at the same time, but from 


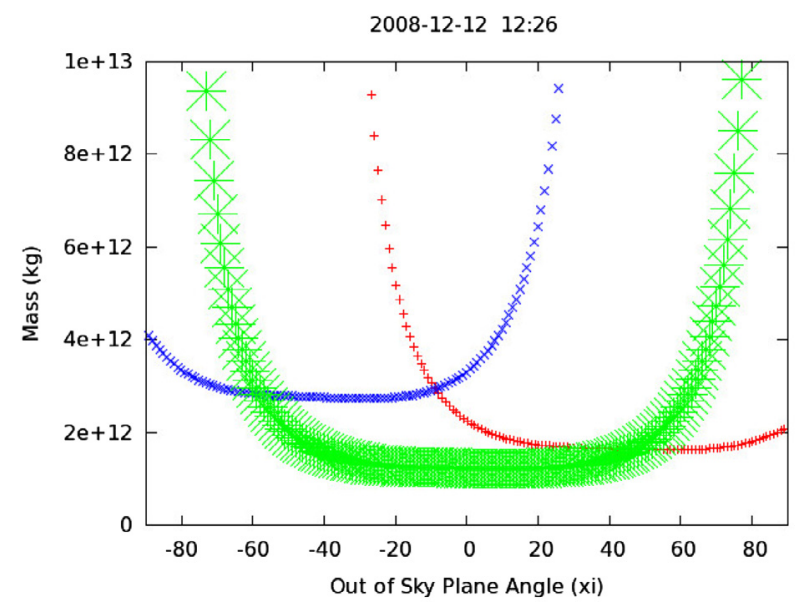

Fig. 7. A mass vs angle from the sky plane $(\xi)$ from three simultaneous images of the same CME, observed on 12 December 2008 at 12:27UT, from SOHO/LASCO-C2 (green), and STEREO-A/ COR2 (red) and -B/COR2 (blue) (C. de Koning, private communication, 2014). The failure of these three plots to intersect at a common point (supposedly representing the "true" location and mass of the CME) demonstrates a possible inadequacy in the calibration of one or more of these coronagraphs.

different observing angles (i.e., from LASCO and COR2-A and -B). Common to each plot is the Thomson plateau (Sect. 4.2) showing a large number of mass measurements at the same value across a large span of $\xi$ values. This demonstrates the robustness of mass measurements but at the cost of the annihilation of information about the 3-D location, as mentioned earlier.

Colaninno \& Vourlidas (2009) devised a 3-D reconstruction technique involving similar plots, which is a potentially robust method if the calibration is sufficiently accurate for each of the different imagers, and the CME and separation between the observers are not excessively large. The plots invariably cross at a particular mass and $\xi$, which is interpreted as the "true" mass and location of the CME. Comparing only the two STEREO results presents this very nicely, with crossover mass and $\xi$ values of $3 \times 10^{12} \mathrm{~kg}$ and $-10^{\circ}$ from the EarthSun sky plane, respectively, for this particular CME. The problem arises when we introduce a third coronagraph, in this case, $\mathrm{LASCO} / \mathrm{C} 2$. There is no common point of intersection between the three curves, and the "true" mass and location of the CME now depends on which combination of imagers we use. There are several possible reasons for this discrepancy; the bulk of the CME mass may not be observable by one or more observers, or we are measuring different areas comprising the CME since its geometry appears different in each image; but the most likely explanation is that one, two, or even all three of the coronagraphs have not been calibrated correctly. It is possible that we could mitigate this discrepancy by adding a correction post-analysis, for example we could have the LASCO plot meet the crossover point between the two CORs by adjusting its radiance such that its mass is tripled, but we would need a substantial investigation to determine whether it is indeed LASCO that requires the correction and not one or both of the CORs.

\section{3-D CME reconstruction: unprecedented data but mixed results}

Sections 3 and 4 describe in detail the obstacles presented to us when attempting to extract meaningful measurements of
CMEs. Many of these are insurmountable, creating a limit in our accuracy (Sect. 6). The arrival of new datasets via the STEREO mission has, however, enabled the development of a few tools that we can utilize to attempt to improve our extraction abilities. As our present review focuses on white light imaging we explore some techniques that have emerged that try to improve our ability to extract meaningful measurements from the "fog" of white light. In recent years these have focused on utilizing the stereoscopic capabilities of STEREO, but have been met with only mixed success. We have found that additional information is available in heliospheric image data that is not available in coronagraphs, as they effectively remove an important impediment imposed upon the latter.

\subsection{3-D reconstruction techniques}

Coronagraphs and heliospheric imagers observe in two dimensions only; what is imaged are the radiances integrated along each line of sight (LOS) through the feature, projected into the plane of the sky of the imager (i.e., the plane for which the LOS vector is normal). The distances and masses determined from these images are projected values and therefore represent a lower limit on the actual parameters. To derive a value that has been corrected for 3-D effects we need to obtain an angle from the sky plane of the measured feature (i.e., its location along the LOS).

Three-dimensional (i.e., "depth") information is not immediately forthcoming in white light images. By far, the most commonly used mode used for the white light images is the unpolarized mode, i.e., the (calibrated) images display the total radiance (defined as $B_{\mathrm{TOT}}=B_{r}+B_{t}=2 B_{t}-p B$, where the $r$ and $t$ subscripts refer to the radial and tangential components of the scattered light respectively (see, e.g., Howard \& Tappin 2009), integrated along the collective lines of sight passing through each pixel. As described further in Section 4.2, the nature of Thomson scattering is such that $B_{\mathrm{TOT}}$ remains almost constant across a large distance from the plane of the sky. This means that the location of a feature along the LOS can vary by a large amount before any noticeable change in its radiance is detected. We must therefore seek alternative means to extract 3-D information about the CME.

The most common means by which workers have attempted to extract 3-D properties is by investigating auxiliary datasets. That is, workers seek datasets that exhibit features known to be related to CMEs, but contain information about their location in 3-D. In-situ measurements provide a great deal of information about a CME passing through a single point a large distance from the Sun, but workers typically seek images of the solar disk to assist in 3-D location. Solar disk images are available through a variety of means, including $\mathrm{H} \alpha$, EUV, and X-rays. Traditionally, one seeks eruptions from the solar "surface" such as flares and eruptive filaments, and active regions. Physically it makes sense to seek these as evidence of the source of the CME eruption, as they arise from magnetically complex regions and exhibit signs of either magnetic reconnection or some other high-energy magnetic field reconfiguration, which probably provided the launch mechanism for the CME. Unfortunately, they serve as poor indicators of CME location for all but the narrowest CMEs, as they tend to occur at only a single footpoint of the CME loop (e.g., Harrison 1986). Other phenomena observed on the solar disk appear to serve as a better proxy for CME location; these include coronal dimming (McIntosh et al. 2007; Reinard \& Biesecker 2009), post-eruptive arcades (Tripathi et al. 2004), and EUV 
waves (e.g., Chen et al. 2005; Attrill et al. 2009). Unfortunately, these are associated with only a small proportion of the CMEs observed in white light and the features best observed on the solar surface (those near disk center) correspond with the white light CMEs that are most difficult to measure (halos).

The STEREO mission (Russell 2008) provided an opportunity for 3-D reconstruction not before available to the solar physics community. STEREO consists of twin spacecraft, oriented into orbits that increase their angular distance from the Sun-Earth line, and each other, with time. This enables simultaneous views of CMEs from separately spaced viewpoints for the first time. Along with an assortment of in-situ, radio, and EUV imaging instruments, both STEREO carry two coronagraphs (COR1 and COR2) and two heliospheric imagers (HI-1 and HI-2), aligned such that their fields of view cover the angular (elongation) range from the Sun to $90^{\circ}$. Following the first attempts of triangulation using COR1 and COR2 observations by Mierla et al. (2008) and Howard \& Tappin (2008), respectively, a large number of 3-D reconstruction techniques using the coronagraphs has emerged in the literature. The earlier reconstruction techniques are reviewed by Mierla et al. (2010) but the number of different techniques continues to grow.

\subsubsection{Using coronagraphs}

The vast majority of publications on the 3-D reconstruction of CMEs from white light images have involved coronagraph data. While the levels of complexity and sophistication vary, the methods for coronagraph reconstruction can be divided into the following three categories:

1. Geometric triangulation of common features observed by each spacecraft (e.g., Howard \& Tappin 2008; Liewer et al. 2009; Temmer et al. 2009; Feng et al. 2012);

2. Volume fitting around the CME envelope, typically using a predetermined configuration and adjusting its geometrical parameters to match that enclosing the CME (e.g., Xie et al. 2004; Thernisien et al. 2006; Boursier et al. 2009a; de Koning et al. 2009);

3. Utilizing polarimetry (e.g., Mierla et al. 2009; Moran et al. 2010; de Koning \& Pizzo 2011).

Geometric triangulation is based on the principle that a feature that appears in multiple observers is at the same location in 3-D space. A worker would select their feature of interest, measure its projected location in each of the coronagraphs (most papers have involved just the two STEREO spacecraft but a few (e.g., Howard \& Tappin 2008; Temmer et al. 2009; Feng et al. 2012) have attempted to achieve this with STEREO and LASCO), and use basic geometry to determine its location in 3-D space. Such a technique is subject to several pitfalls, including the assumptions that the measured feature is sufficiently spatially localized, that it is the same feature as that observed with the other imagers and that the same part of the feature is being measured, and whether the instruments are sufficiently calibrated to identify the same feature in the same location.

Volume fitting assumes that the CME lies within a particular volume and adjusts the parameters of that volume to fit the observed geometry of the CME. The selected volume ranges in complexity, including a simple polygon (e.g., de Koning et al. 2009) to a cone (e.g., Xie et al. 2004) to a loop-like configuration (e.g., Thernisien et al. 2006). Such techniques are appropriate for simple identification of the general volume in which the CME lies, but do not contain measurements of high levels of accuracy.

Polarimetry enables the utilization of a property of Thomson scattering not available through unpolarized light. As shown in Figure 5b, the Thomson plateau (Sect. 4.2) is not present when observing the polarized radiance $p B$. The problem of non-localization along the LOS is therefore somewhat mitigated; in other words, a feature cannot move as far from the plane of the sky before its observed radiance is diminished by a detectable quantity. By comparing the unpolarized with the polarized radiance we can therefore identify the distance from the plane of the sky of the feature along any given LOS. This enables the construction of the 3-D CME, pixel-by-pixel. The scattered radiance distribution is symmetric about the sky plane in coronagraphs, and so this technique alone cannot distinguish whether the feature is ahead of or behind the sky plane. Hence, the technique is generally used with another that enables this determination. For example, one method involves combining the volume fitting technique of de Koning et al. (2009) with the polarimetry technique of de Koning \& Pizzo (2011).

Despite the large number of papers that have been published describing methods of 3-D reconstruction, no successful method has yet emerged to accurately reconstruct the CME from coronagraph images alone. The volume fitting and polarimetry techniques described in the previous paragraph appear to be the most consistently successful, but they yield only a "hazy bubble" structure for the CME (see, e.g., Fig. 6 of de Koning \& Pizzo 2011). The reasons that these new datasets have had so little impact are because of the physical limitations inherent in the instrumental measuring process, which are discussed in Sections 3 and 4.

\subsubsection{Using heliospheric imagers}

Heliospheric imagery involves the same principles as coronagraphs: they observe Thomson scattered white light and are therefore subject to the same limitations that I have explored throughout this review. Likewise the 3-D reconstruction techniques applied to heliospheric imagers have been limited in their success. Geometrical triangulation is nonsense at the distances observed by heliospheric imagers as it is impossible to identify a sufficiently localized feature with which to perform triangulation that is in any way reliable (Howard 2011b), and polarimetry is not available as no contemporary heliospheric imager has a polarizing capability. This leaves us with the volume fitting technique, but here the heliospheric imagers have an advantage over coronagraphs. Since they observe across a very large angular extent, we have the ability to extract 3-D geometrical information that is not available in coronagraphs.

Howard (2011b) provides a review on the enhanced abilities of heliospheric imagers for 3-D information extraction. Briefly, two important assumptions that are applied for the analysis of coronagraph images - that the angles of all the observed features from the Sun are small and that the features are close to the sky plane - break down at larger angles from the Sun. Also the geometry of the CME itself, which can be regarded as sufficiently small to be ineffective at small angles, governs its appearance (e.g., the location of the leading edge), particularly if the CME is large. This necessitates a full geometrical reconstruction of the CME, similarly to the volume 
fitting technique used for coronagraphs, but taking advantage of the physics of Thomson scattering at large angles from the Sun. As with those developed for coronagraphs, techniques of varying complexity and sophistication have been developed. Some noteworthy examples include Tappin \& Howard (2009), Wood \& Howard (2009), Lugaz et al. (2010), Byrne et al. (2010), and Möstl \& Davies (2013).

Finally, I would like to comment on the potential of polarized heliospheric imagery. The author and colleagues have developed the theory demonstrating this ability (DeForest et al. 2013b; Howard et al. 2013a) and explored the feasibility of using data processing to extract features from the images and measure them (DeForest et al., 2011 2012, 2013a; Howard et al. 2012). Additionally, we performed some preliminary studies using simulated polarized heliospheric imagers (Howard et al. 2013a) and found that, just as in the case of coronagraphs, additional 3-D information can be extracted from heliospheric imagers. Further, we have investigated the feasibility of identifying CMEs in significantly degraded HI-2 images, to explore the quality of the data needed to extract meaningful measurements. DeForest \& Howard (2015) found that CMEs were detectable using data that was so poor in quality that they could have been measured from the ground. These studies, combined with the geometric advantages of heliospheric imagers over coronagraphs, enable us to increase the accuracy of 3-D reconstruction beyond the capabilities of coronagraphs. Hence the next generation of heliospheric imagers should be one capable of polarimetry. Such a capability will not be present in those heliospheric imagers currently under development for the Solar Probe Plus or Solar Orbiters, but are under consideration for a separate mission being proposed for placement into an L5 Lagrange point orbit.

\subsection{Putting it all together}

The complete picture of CME reconstruction requires utilization of all of the observing datasets at our disposal, including solar imagers such as magnetograms, $\mathrm{H} \alpha, \mathrm{EUV}$, and X-ray, white light imagers, in-situ, and radio. Even then we need to be fortunate enough such that all of the instruments are observing in the right place at the right time and for the CME to be directed in such a way as to optimize their measurement capabilities. To understand the physics involved we also need modeling, both empirical and theoretical. The bounds lie in the data, as our ability to extract the information we need is hampered here as we have explored exhaustively in this review. Consequently, very few serious efforts have been made to physically reconstruct the CMEs using data alone.

Recent work by Howard \& DeForest (2014) took advantage of a unique period in the STEREO mission when both STEREO were at quadrature with each other, and around $45^{\circ}$ from the Sun-Earth line. During this time (December 2008), a CME erupted that was associated with a magnetic flux rope aligned approximately east-west and spanning some $90^{\circ}$ across the Sun. This orientation enabled the viewing of the CME roughly tangential and normal to the flux rope axis, allowing the observation of its central structure as well as its cross-section. By combining the white light data with the EUV solar disk images, as well as the in-situ measurements, we were able to reconstruct the CME flux rope and develop a narrative for its launch and departure from the corona.

Figure 8 shows a simplified cartoon of the flux rope launch and its configuration near $1 \mathrm{AU}$. We constructed a narrative describing an asymmetric launch not unlike the scenario shown in Figure 6b, where the western end launched first and the rest followed in a manner perhaps analogous to a strip of carpet being torn from a floor. We note that the single flux rope was subject to three different onset mechanisms (tether cutting, following by mass draining, followed by kink instability) and a possible bisection before completely departing from the Sun.

The CME that is associated with this flux rope was the first geoeffective CME observed by STEREO and has been reported by many workers (e.g., Davis et al. 2009; Byrne et al. 2010; Liu et al. 2010; Panasenco et al. 2011; Howard \& DeForest 2012a). The CME as it appeared in coronagraphs consisted of multiple loops enclosed within an overlying bubble (see Fig. 8a of Howard \& DeForest 2014). While the loops appeared separate from each other, we interpreted them as manifestations of the same flux rope; we observe different cross-sections at different "depths" in the coronagraph, as the flux rope erupts asymmetrically. Several hours later a completely separate structure was observed in the coronagraphs, appearing as little more than a puff of increased density (see Fig. 8b of Howard \& DeForest 2014). This was the signature of the departure of the final segment of the flux rope.

For us to produce this narrative we relied on sophisticated processing of the white light data, careful observation and tracking of features in the EUV images, utilization of the in-situ datasets (see DeForest et al. 2013a), knowledge of the various onset mechanisms that have been modeled for CMEs, and some luck as to have the STEREO spacecraft in the right viewpoints for optimally observing this particular flux rope. It is this kind of effort that will be required for the development of future CME geometry and eruption narrative reconstruction.

\section{Discussion}

The purpose of this review is to highlight the difficulties associated with the detection and measurement of CMEs. I have focused primarily on white light (coronagraph and heliospheric imager) detection, as they can be regarded as the binding agents between the various solar observations and the in-situ measurements of CMEs. I have explored the progress in CME measurement over the decades and described in detail those factors that impede our ability to extend our measuring capabilities further.

Despite the ongoing efforts by many workers, we may need to conclude that the accuracy of CME measurements cannot be improved upon with the current generation of white light imagers. This is not a spatial resolution, cadence, or even sensitivity issue, it is simply that there is likely no more information available in these images. The factors explored in this review (optical thinness, geometry, Thomson scattering) leave us with the ability to reconstruct the CME as only a hazy mass, given the current generation of white light imagers. This is probably sufficient for operational efforts such as space weather forecasting, as the important factors needed there (leaving aside magnetic field information that is not available in white light imagers) are whether the mass is directed toward Earth and when we can expect it to arrive there. However, for measurements of accuracy required to advance our scientific knowledge of CMEs we need to extend beyond the current generation of imagers. Polarimetry is not yet a fully explored area of white light imaging and it does offer some promise for advancement, particularly in heliospheric imaging, and these efforts need to continue to be developed. 


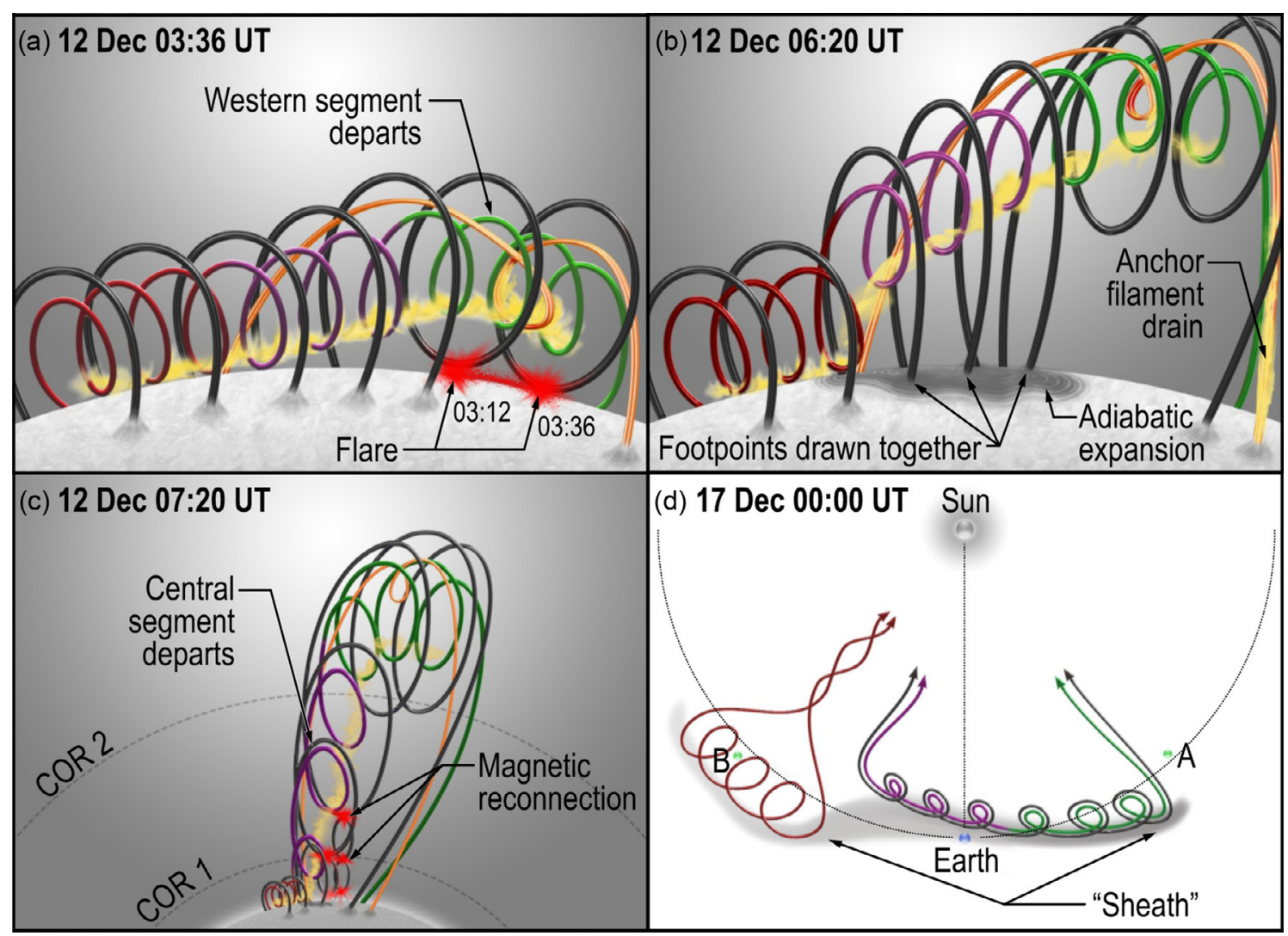

Fig. 8. A selection of four panels from Figure 9 of Howard \& DeForest (2014) presenting a basic narrative of the launch of a flux rope that gave rise to the CME on 12 December 2008. The narrative was constructed via careful observation and measurement of features observed with white light and EUV imagery and in-situ spacecraft. (a) The initial launch caused by the magnetic reconnection of the overlying field at the western end, manifest by a flare, (b) the departure of the central segment of the flux rope by means of mass draining and the stretching of the overlying field, (c) the departure of the field overlying the central segment by magnetic reconnection, (d) an illustration of an estimated configuration of the flux rope when it arrived at the in-situ spacecraft near $1 \mathrm{AU}$.

Those CMEs that are of most interest to space weather are typically the large energetic ones. These are often observed by white light imagers in the classic three-part configuration. What we observe in white light is a 2-D manifestation of the entire 3-D configuration, combining the integrated lines of sight, all folded into an image on the sky plane. Threedimensional information is not directly available here, but we can employ auxiliary datasets and models to help us reconstruct the physics involved in the eruption of the CME structure. We must all get into the mindset of regarding CMEs as two-dimensional projections of a collective of lines of sight through a 3-D object. We must regard projection and simultaneous activity in the foreground and background with each observation. Often, what appears to be a revealing new physical property of CMEs may simply be a projection effect.

\subsection{Concluding remarks}

Following such notes of caution one may wonder what the best course of action for advancing our ability is to extract scientific information about CMEs. We have exhausted the information we can extract using unpolarized white light imagery, but further information is possibly still available using polarimetry. The design of the next generation of white light imagers should include a polarizing ability and any novel means by which such information can be optimally made available. A further path may involve spectroscopy across a wide FOV, such as the ASPIIC coronagraph (Lamy et al. 2010) under development for the Proba-3 mission. Beyond this we must coordinate our efforts, combining multiple observations of the Sun, corona, and heliosphere with modeling and carefully reconstruct the physical narrative of the lifetime of the CME. We must regard CMEs as extended 3-D objects and that the importance of perspective cannot be understated. Finally, we must be careful in the language we use to describe CMEs and their related features. Too often are terms like "CME", "source", "brightness", and "flux rope" used without a clear definition or even ambiguously. Given how some of the terms we use are poorly defined to begin with, it is not a bad idea to include a section in our papers defining the terms we will be using throughout.

Acknowledgements. Work for this paper was supported by the NASA GI Grant NNX13AE01G, the NSF SHINE competition (Grant 1260321), and the AFOSR (Grant 14RT0429). The editor thanks two anonymous referees for their assistance in evaluating this paper.

\section{References}

Antiochos, S.K., C.R. DeVore, and J.A. Klimchuk. A model for solar coronal mass ejections. Astrophys. J., 510, 485-493, 1999, DOI: $10.1086 / 30656$.

Athay, R.G., and R.M.E. Illing. Analysis of the prominence associated with the coronal mass ejection of August 18 . J. Geophys. Res., 91, 10961-10973, 1980, DOI: 10.1029/JA091iA10p10961.

Attrill, G.D.R., A.J. Engell, M.J. Wills-Davey, P. Grigis, and P. Testa. Hinode/XRT and STEREO observations of a diffuse coronal "wave"-coronal mass ejection-dimming event. Astrophys. J., 704, 1296, 2009, DOI: 10.1088/0004-637X/704/2/1296. 
Bao, X., H. Zhang, and J. Lin. Four coronal mass ejections and their associated surface activity observed on 26 October 2003. In: K.P. Dere, J. Wang, and Y. Yan, Editors, Proc. IAU Symp., Coronal and Stellar Mass Ejections, St. Petersburg, Russia, 223-224, 2005, DOI: 10.1017/S174392130500058X.

Bewsher, D., D.S. Brown, C.J. Eyles, B.J. Kellett, G.J. White, and B. Swinyard. Determination of the photometric calibration and large-scale flatfield of the STEREO heliospheric imagers: I. HI-1. Sol. Phys., 264, 433-460, 2010, DOI: 10.1007/s11207-010-9582-8.

Billings, D.E. A Guide to the Solar Corona, Academic Press, New York, 1966

Bothmer, V., and R. Schwenn. The structure and origin of magnetic clouds in the solar wind. Ann. Geophys., 16, 1, 1998, DOI: 10.1007/s00585-997-0001-x.

Boursier, Y., P. Lamy, and A. Llebaria. Three-dimensional kinematics of coronal mass ejections from STEREO/SECCHI-COR2 observations in 2007-2008. Sol. Phys., 256, 131-147, 2009a, DOI: $10.1007 / \mathrm{s} 11207-009-9358-1$.

Boursier, Y., P. Lamy, A. Llebaria, F. Goudail, and S. Robelus. The ARTEMIS catalog of LASCO coronal mass ejections. Sol. Phys., 257, 125-147, 2009b, DOI: 10.1007/s11207-009-9370-5.

Brueckner, G.E., R.A. Howard, M.J. Koomen, C.M. Korendyke, D.J. Michels, et al. The Large Angle Spectroscopic Coronagraph (LASCO). Sol. Phys., 162, 357-402, 1995, DOI: $10.1007 / \mathrm{BF} 00733434$

Burlaga, L., E. Sittler, F. Mariani, and R. Schwenn. Magnetic loop behind an interplanetary shock - Voyager, Helios, and IMP 8 observations. J. Geophys. Res., 86, 6673-6684, 1981, DOI: 10.1029/JA086iA08p06673.

Byrne, J.P., S.A. Maloney, R.T.J. McAteer, J.M. Refojo, and P.T. Gallagher. Propagation of an Earth-directed coronal mass ejection in three dimensions. Nature Comm., 1, 74, 2010, DOI: $10.1038 /$ ncomms 1077 .

Byrne, J.P., H. Morgan, S.R. Shadia, and P.T. Gallagher. Automatic detection and tracking of coronal mass ejections, II. Multiscale filtering of coronagraph images. Astrophys. J., 752, 145, 2012, DOI: $10.1088 / 0004-637 \mathrm{X} / 752 / 2 / 145$.

Cane, H.V., S.W. Kahler, and N.R. Sheeley Jr. Interplanetary shocks preceded by solar filament eruptions. J. Geophys. Res., 91, 13321-13329, 1986, DOI: 10.1029/JA091iA12p13321.

Chen, P.F. Coronal mass ejections: models and their observational basis. Living Rev. Sol. Phys., 8, 1, 2011, DOI: $10.12942 /$ lrsp-2011-1.

Chen, P.F., M.D. Ding, and C. Fang. Synthesis of CME-associated Moreton and EIT wave features from MHD simulations. Space Sci. Rev., 121, 201-211, 2005, DOI: 10.1007/s11214-006-3911-0.

Colaninno, R.C., and A. Vourlidas. First determination of the true mass of coronal mass ejections: a novel approach to using the two STEREO viewpoints. Astrophys. J., 698, 852-858, 2009, DOI: $10.1088 / 0004-637 \mathrm{X} / 698 / 1 / 852$

Crooker, N.U. Solar and heliospheric geoeffective disturbances. J. Atmos. Sol. Terr. Phys., 62, 1071-1085, 2000, DOI: 10.1016/S1364-6826(00)00098-5.

Crooker, N.U., J.T. Gosling, E.J. Smith, and C.T. Russell. A bubblelike coronal mass ejection flux rope in the solar wind. $A G U$ Geophys. Monograph, 58, 365-371, 1990.

Davis, C.J., J.A. Davies, M. Lockwood, A.P. Rouillard, C.J. Eyles, and R.A. Harrison. Stereoscopic imaging of an Earth-impacting solar coronal mass ejection: a major milestone for the STEREO mission. Geophys. Res. Lett., 36, L08102, 2009, DOI: $10.1029 / 2009$ GL038021.

DeForest, C.E., and T.A. Howard. Feasibility of heliospheric imaging from near Earth. Astrophys. J., 804, 126, 2015, DOI: $10.1088 / 0004-637 \mathrm{X} / 804 / 2 / 126$

DeForest, C.E., T.A. Howard, and S.J. Tappin. Observations of detailed structure in the solar wind at $1 \mathrm{AU}$ with STEREO/HI-2. Astrophys. J., 738, 103, 2011, DOI: 10.1088/0004-637X/738/1/103.

DeForest, C.E., T.A. Howard, and D.J. McComas. Disconnecting open solar magnetic flux. Astrophys. J., 745, 36, 2012, DOI: $10.1088 / 0004-637 \mathrm{X} / 745 / 1 / 36$.
DeForest, C.E., T.A. Howard, and D.J. McComas. Tracking coronal features from the low corona to Earth: a quantitative analysis of the 2008 December 12 coronal mass ejection. Astrophys. J., 769, 43, 2013a, DOI: 10.1088/0004-637X/769/1/43.

DeForest, C.E., T.A. Howard, and S.J. Tappin. The Thomson surface: II. Polarization. Astrophys. J., 765, 44, 2013b, DOI: $10.1088 / 0004-637 X / 765 / 1 / 44$.

de Koning, C.A., and V.J. Pizzo. Polarimetric localization: a new tool for calculating the CME speed and direction of propagation in near-real time. Space Weather, 9, S03001, 2011, DOI: 10.1029/2010SW000595.

de Koning, C.A., V.J. Pizzo, and D.A. Biesecker. Geometric localization of CMEs in 3D space using STEREO beacon data: first results. Sol. Phys., 256, 167-181, 2009, DOI: $10.1007 / \mathrm{s} 11207-009-9344-7$.

DeMastus, H.L., W.J. Wagner, and R.D. Robinson. Coronal disturbances, I: fast transient events observed in the green coronal emission line during the last solar cycle. Sol. Phys., 31, 449-459, 1973, DOI: 10.1007/BF00152820.

Dryer, M. Interplanetary shock waves generated by solar flares. Space Sci. Rev., 15, 403-468, 1974, DOI: 10.1007/BF00178215.

Fan, Y., and S.E. Gibson. The emergence of a twisted magnetic flux tube into a preexisting coronal arcade. Astrophys. J., 589, L105-L108, 2003, DOI: 10.1086/375834.

Fan, Y., and S.E. Gibson. Numerical simulations of threedimensional coronal magnetic fields resulting from the emergence of twisted magnetic flux tubes. Astrophys. J., 609, 1123-1133, 2004, DOI: 10.1086/421238.

Farrugia, C.J., H. Matsui, H. Kuchaerek, R.B. Torbert, C.W. Smith, et al. Interplanetary coronal mass ejection and ambient interplanetary field correlations during the Sun-Earth connection events of October-November 2003. J. Geophys. Res., 110, A09S13, 2005, DOI: 10.1029/2004JA010968.

Feng, L., B. Inhester, Y. Wei, W.Q. Gan, T.L. Zhang, and M.Y. Wang. Morphological evolution of a three-dimensional coronal mass ejection cloud reconstructed from three viewpoints. Astrophys. J., 751, 18, 2012, DOI: 10.1088/0004-637X/751/1/18.

Forsyth, R.J., V. Bothmer, C. Cid, N.U. Crooker, T.S. Horbury, et al. ICMEs in the inner heliosphere: origin, evolution and propagation effects. Report of working group G. Space Sci. Rev., 123, 383-416, 2006, DOI: 10.1007/s11214-006-9022-0.

Frazin, R.A., A.M. Vásquez, W.T. Thompson, R.J. Hewett, P. Lamy, A. Llebaria, A. Vourlidas, J. Burkepile. Intercomparison of the LASCO-C2, SECCHI-COR1, SECCHI-COR2, and Mk4 coronagraphs. Sol. Phys., 280, 273-293, 2012, DOI: $10.1007 / \mathrm{s} 11207-012-0028-3$.

Fuller, J., S.E. Gibson, G. de Toma, and Y. Fan. Observing the unobservable? Modeling coronal cavity densities. Astrophys. J., 678, 515-530, 2008, DOI: 10.1086/533527.

Gopalswamy, N., S. Yashiro, Y. Liu, G. Michalek, A. Vourlidas, M.L. Kaiser, and R.A. Howard. Coronal mass ejections and other extreme characteristics of the 2003 October-November solar eruptions. J. Geophys. Res., 110, A09S15, 2005, DOI: 10.1029/2004JA010958.

Gosling, J.T. The solar flare myth. J. Geophys. Res., 98, 18937-18949, 1993, DOI: 10.1029/93JA01896.

Gosling, J.T., E. Hildner, R.M. MacQueen, R.H. Munro, A.I. Poland, and C.L. Ross. Mass ejections from the Sun - a view from SKYLAB. J. Geophys. Res., 79, 4581-4587, 1974, DOI: $10.1029 / \mathrm{JA} 079 \mathrm{i} 031 \mathrm{p} 04581$.

Gosling, J.T., E. Hildner, R.M. MacQueen, R.H. Munro, A.I. Poland, and C.L. Ross. Direct observations of a flare related coronal and solar wind disturbance. Sol. Phys., 40, 439-448, 1975, DOI: $10.1007 / \mathrm{BF} 00162390$

Gosling, J.T., E. Hildner, R.M. MacQueen, R.H. Munro, A.I. Poland, and C.L. Ross. The speeds of coronal mass ejections events. Sol. Phys., 48, 389-397, 1976, DOI: $10.1007 / \mathrm{BF} 00152004$

Harrison, R.A. Solar coronal mass ejections and flares. Astron. Astrophys., 162, 283-291, 1986. 
Hildner, E., J.T. Gosling, R.M. MacQueen, R.H. Munro, A.I. Poland, and C.L. Ross. The large coronal transient of 10 June 1973, I: observational description. Sol. Phys., 42, 163-177, 1975, DOI: 10.1007/BF00153293.

Howard, R.A., M.J. Koomen, D.J. Michels, R. Tousey, C.R. Dewiler, et al. Synoptic observations of the solar corona during Carrington rotations 1580-1596 (11 October 1971-15 January 1973), World Data Center A for Solar-Terr. Phys, Report UAG 48A, 1976.

Howard, R.A., D.J. Michels, N.R. Sheeley Jr., and M.J. Koomen. The observation of a coronal transient directed at Earth. Astrophys. J. Lett., 263, L101-L104, 1982, DOI: 10.1086/183932.

Howard, R.A., N.R. Sheeley Jr., D.J. Michels, and M.J. Koomen, Coronal mass ejections - 1979-1981, J. Geophys. Res., 90, 8173-8191, 1985, DOI: 10.1029/JA090iA09p08173.

Howard, T. Coronal mass ejections: An introduction, Astrophysics and Space Science Library, 376, Springer, New York, 2011a, DOI: $10.1007 / 978-1-4419-8789-1$.

Howard, T. Three-dimensional reconstruction of coronal mass ejections using heliospheric imager data. J. Atmos. Sol. Terr. Phys., 73, 1242-1253, 2011b, DOI: 10.1016/j.jastp.2010.08.009.

Howard, T. Space weather and coronal mass ejections, SpringerBriefs in Astronomy, XIII, ISBN: 978-1-4614-7975-8, 2014.

Howard, T. Measuring an eruptive prominence at large distances from the Sun: I. Ionization and early evolution. Astrophys. J., 806, 175, 2015a, DOI: 10.1088/0004-637X/806/2/175.

Howard, T. Measuring an eruptive prominence at large distances from the Sun: II. Approaching 1 AU. Astrophys. J., 806, 176, 2015b, DOI: 10.1088/0004-637X/806/2/176.

Howard, T.A., and C.E. DeForest. Inner heliospheric flux rope evolution via imaging of coronal mass ejections. Astrophys. J., 746, 64, 2012a, DOI: 10.1088/0004-637X/746/1/64.

Howard, T.A., and C.E. DeForest. The Thomson surface: I. Reality and myth. Astrophys. J., 752, 130, 2012b,

DOI: $10.1088 / 0004-637 \mathrm{X} / 752 / 2 / 130$.

Howard, T.A., and C.E. DeForest. The formation and launch of a coronal mass ejection flux rope: a narrative based on observations. Astrophys. J., 796, 33, 2014, DOI: 10.1088/0004-637X/796/1/33.

Howard, T.A., and S.J. Tappin. Three-dimensional reconstruction of two solar coronal mass ejections using the STEREO spacecraft. Sol. Phys., 252, 373-383, 2008, DOI: 10.1007/s11207-008-9262-0.

Howard, T.A., and S.J. Tappin. Interplanetary coronal mass ejections observed in the heliosphere: 1. Review of theory. Space Sci. Rev., 147, 31-54, 2009, DOI: 10.1007/s11214-009-9542-5.

Howard, T.A., C.E. DeForest, and A.A. Reinard. White light observations of solar wind transients and comparison with auxiliary datasets. Astrophys. J., 754, 102, 2012, DOI: $10.1088 / 0004-637 X / 754 / 2 / 102$.

Howard, T.A., S.J. Tappin, D. Odstrcil, and C.E. DeForest. The Thomson surface: III, Tracking features in 3-D. Astrophys. J., 765, 45, 2013a, DOI: $10.1088 / 0004-637 X / 765 / 1 / 45$.

Howard, T.A., M.M. Bisi, A. Buffington, J.M. Clover, M.P. Cooke, et al. The Solar Mass Ejection Imager and its heliospheric imaging legacy. Space Sci. Rev., 180, 1-38, 2013b, DOI: $10.1007 / \mathrm{s} 11214-013-9992-7$.

Hundhausen, A.J., J.T. Burkepile, and O.C. St. Cyr. Speeds of coronal mass ejections: SMM observations from 1980 and 1984-1989. J. Geophys. Res., 99, 6543-6552, 1994, DOI: 10.1029/93JA03586.

Illing, R.M.E., and A.J. Hundhausen. Observation of a coronal transient from 1.2 to 6 solar radii. J. Geophys. Res., 90, 275-282, 1985, DOI: 10.1029/JA090iA01p00275.

Illing, R.M.E., and R.G. Athay. Physical conditions in eruptive prominences at several solar radii. Sol. Phys., 105, 173-190, 1986, DOI: $10.1007 / \mathrm{BF} 00156385$.

Klein, L.W., and L.F. Burlaga. Interplanetary magnetic clouds at 1 AU. $J$. Geophys. Res., 87, 613-624, 1982, DOI: 10.1029/JA087iA02p00613.

Koomen, M.J., C.R. Detwiler, G.E. Brueckner, H.W. Cooper, and R. Tousey. White light coronagraph in OSO-7. Appl. Opt., 14, 743-751, 1975, DOI: 10.1364/AO.14.000743.
Lamy, P., L. Damé, S. Vivès, and A. Zhukov. ASPIICS: a giant coronagraph for the ESA/PROBA-3 formation flying mission. Proc. SPIE, 7731, 773118, 2010, DOI: 10.1117/12.858247.

Lepping, R.P., L.F. Burlaga, and J.A. Jones. Magnetic field structure of interplanetary magnetic clouds at 1 AU. J. Geophys. Res., 95, 11957-11965, 1990, DOI: 10.1029/JA095iA08p11957.

Liewer, P.C., E.M. de Jong, J.R. Hall, R.A. Howard, W.T. Thompson, J.L. Culhane, L. Bone, and L. van Driel-Gesztelyi. Stereoscopic analysis of the 19 May 2007 erupting filament. Sol. Phys., 256, 57-72, 2009, DOI: 10.1007/s11207-009-9363-4.

Liu, Y., A. Thernisien, J.G. Luhmann, A. Vourlidas, J.A. Davies, R.P. Lin, and S.D. Bale. Reconstructing coronal mass ejections with coordinated imaging and in situ observations: global structure, kinematics, and implications for space weather forecasting. Astrophys. J., 722, 1762-1777, 2010, DOI: $10.1088 / 0004-637 \mathrm{X} / 722 / 2 / 1762$.

Low, B.C. The nature of the quiescent prominence cavity. Bull. Amer. Astron. Soc., 25, 1218, 1993.

Lugaz, N., J.N. Hernandez-Charpak, I.I. Rousev, C.J. Davis, A. Vourlidas, and J.A. Davis. Determining the azimuthal properties of coronal mass ejections from Multi-spacecraft remove-sensing observations with STEREO SECCHI. Astrophys. J., 715, 493-499, 2010, DOI: $10.1088 / 0004-637 X / 715 / 1 / 493$.

Lynch, B.J., S.K. Antiochos, C.R. DeVore, J.G. Luhmann, and T.H. Zurbuchen. Topological evolution of a fast magnetic breakout CME in three dimensions. Astrophys. J., 683, 1192-1206, 2008, DOI: $10.1086 / 589738$.

MacQueen, R.M. Coronal transients: a summary. Philos. Trans. R. Soc. London, 297, 605-620, 1980.

MacQueen, R.M., J.A. Eddy, J.T. Gosling, E. Hildner, R.H. Munro, G.A. Newkirk Jr., A.I. Poland, and C.L. Ross. The outer solar corona as observed from Skylab: preliminary results. Astrophys. J., 187, L85, 1974, DOI: 10.1086/181402.

MacQueen, R.M., A. Csoeke-Poeckh, E. Hildner, L. House, R. Reynolds, A. Stanger, H. Tepoel, and W. Wagner. The high altitude observatory coronagraph/polarimeter on the solar maximum mission. Sol. Phys., 65, 91-107, 1980, DOI: $10.1007 / \mathrm{BF} 00156878$.

Malandraki, O.E., D. Lario, L.J. Lanzerotti, E.T. Sarris, A. Geranios, and G. Tsiropoula. October/November 2003 interplanetary coronal mass ejections: ACE/EPAM solar energetic particle observations. J. Geophys. Res., 110, A09S06, 2005, DOI: $10.1029 / 2004 J A 010926$.

Manchester IV, W.B., A. Vourlidas, G. Toth, N. Lugaz, I.I. Roussev, T.I. Gombosi, I. Sokolov, D. De Zeeuw, and M. Opher. Threedimensional MHD simulation of the 2003 October 28 coronal mass ejection: comparison with LASCO coronagraph observations. Astrophys. J., 684, 1448-1460, 2008, DOI: $10.1086 / 590231$.

Martin, S.F., S.H.B. Livi, and J. Wang. The cancellation of magnetic flux. II - in a decaying active region. Australian J. Phys., 38, 929-959, 1985.

McIntosh, S.W., R.J. Leamon, A.R. Davey, and M.J. Wills-Davey. The posteruptive evolution of a coronal dimming. Astrophys. J., 660, 1653-1659, 2007, DOI: 10.1086/512665.

Michels, D.J., R.A. Howard, M.J. Koomen, and N.R. Sheeley Jr. Satellite observations of the outer corona near sunspot maximum. In: M.R., Kundu, and T.E. Gergely, Editors, Radio physics of the Sun, D. Reidel, Hingham, MA, 439, 1980.

Mierla, M., J. Davila, W. Thompson, B. Inhester, N. Srivastava, M. Kramar, O.C. St. Cyr, G. Stenborg, and R.A. Howard. A quick method for estimating the propagation direction of coronal mass ejection using STEREO-COR1 images. Sol. Phys., 252, 385-396, 2008, DOI: $10.1007 / \mathrm{s} 11207-008-9267-8$.

Mierla, M., B. Inhester, C. Marqué, L. Rodriguez, S. Gissot, A.N. Zhukov, D. Berghmans, and J. Davila. On 3D reconstruction of coronal mass ejections: I. Method description and application to SECCHI-COR data. Sol. Phys., 259, 123-141, 2009, DOI: $10.1007 / \mathrm{s} 11207-009-9416-8$. 
T.A. Howard: The detection and measurement of coronal mass ejections

Mierla, M., B. Inhester, A. Antunes, Y. Boursier, J.P. Byrne, et al. On the 3-D reconstruction of coronal mass ejections using coronagraph data. Ann. Geophys., 28, 203-215, 2010, DOI: 10.5194/angeo-28-203-2010.

Mierla, M., I. Chifu, B. Inhester, L. Rodriguez, and A. Zhuov. Low polarised emission from the core of coronal mass ejections. Astron. Astrophys., 530, L1, 2011, DOI: 10.1051/0004-6361/201016295.

Minnaert, M., On the continuous spectrum of the corona and its polarisation, Z. Astrophys., 1, 209-236, 1930.

Moore, R.L., A.C. Sterling, H.S. Hudson, and J.R. Lemen. Onset of the magnetic explosion in solar flares and coronal mass ejections. Astrophys. J., 552, 833-848, 2001, DOI: 10.1086/320559.

Moran, T.G., J.M. Davila, and W.T. Thompson. Three-dimensional polarimetric coronal mass ejection localization tested through triangulation. Astrophys. J., 712, 453-458, 2010,

DOI: $10.1088 / 0004-637 X / 712 / 1 / 453$.

Morrill, J.S., C.M. Korendyke, G.E. Brueckner, F. Giovane, R.A. Howard, et al. Calibration of the SOHO/LASCO C3 white light coronagraph. Sol. Phys., 233, 331-372, 2006, DOI: $10.1007 / \mathrm{s} 11207-006-2058-1$.

Möstl, C., and J.A. Davies. Speeds and arrival times of solar transients approximated by self-similar expanding circular fronts. Sol. Phys., 285, 411-423, 2013, DOI: 10.1007/s11207-012-9978-8.

Poland, A.I., and R.H. Munro. Interpretation of broad-band polarimetry of solar coronal transients - importance of H-alpha emission. Astrophys. J., 209, 927-934, 1976, DOI: $10.1086 / 154791$

Poland, A.I., R.A. Howard, M.J. Koomen, D.J. Michels, and N.R. Sheeley Jr. Coronal transients near sunspot maximum. Sol. Phys., 69, 169-175, 1981, DOI: 10.1007/BF00151264.

Panasenco, O., S. Martin, A.D. Joshi, and N. Srivastava. Rolling motion in erupting prominences observed by STEREO. J. Atmos. Sol. Terr. Phys., 73, 1129-1137, 2011,

DOI: $10.1016 /$ j.jastp.2010.09.010.

Rachmeler, L.A., C.E. DeForest, and C.C. Kankelborg. Reconnectionless CME eruption: putting the Aly-Sturrock conjecture to rest. Astrophys. J., 693, 1431-1436, 2009,

DOI: $10.1088 / 0004-637 X / 693 / 2 / 1431$.

Reinard, A.A., and D.A. Biesecker. The relationship between coronal dimming and coronal mass ejection properties. Astrophys. J., 705, 914, 2009, DOI: 10.1088/0004-637X/705/1/91.

Robbrecht, E., and D. Berghmans. Automated recognition of coronal mass ejections (CMEs) in near-real-time data. Astron Astrophys., 425, 1097-1106, 2004, DOI: 10.1051/0004-6361:20041302.

Russell, C. Editor, The STEREO mission. Space Sci. Rev., 136, 1-3, 2008.

Rust, D.M., E. Hildner, M. Dryer, A.N. McClymont, S.M.P. McKenna-Lawlor, et al. Mass ejections, in Solar flares: $A$ monograph from Skylab Solar Workshop II, Colorado Associated University Press, 273-339, 1980

Schmahl, E., and E. Hildner. Coronal mass ejections-kinematics of the 19 December 1973 event. Sol. Phys., 55, 473-490, 1977, DOI: $10.1007 / \mathrm{BF} 00152588$

Schuster, A. On the polarisation of the solar corona. M. Not. R. Astron. Soc., 40, 35-57, 1879.

Sturrock, P.A. The role of eruption in solar flares. Sol. Phys., 121, 387-397, 1989, DOI: 10.1007/BF00161708.
Sun, X., J.T. Hoeksema, Y. Liu, T. Wiegelmann, and K. Hayashi. Evolution of magnetic field and energy in a major eruptive active region based on SDO/HMI observation. Astrophys. J., 748, 77, 2012, DOI: 10.1088/0004-637X/748/2/77.

Tappin, S.J., and T.A. Howard. Interplanetary coronal mass ejections observed in the heliosphere: 2. Model and data comparison. Space Sci. Rev., 147, 55-87, 2009, DOI: $10.1007 / \mathrm{s} 11214-009-9550-5$.

Tappin, S.J., T.A. Howard, M.M. Hampson, R.N. Thompson, and C.E. Burns. On the autonomous detection of coronal mass ejections in heliospheric imager data. J. Geophys. Res., 117, A05103, 2012, DOI: 10.1029/2011JA017439.

Temmer, M., S. Preiss, and A.M. Veronig. CME project effects studied with STEREO/COR and SOHO/LASCO. Sol. Phys., 256, 183-199, 2009, DOI: 10.1007/s11207-009-9336-7.

Thernisien, A.F.R., R.A. Howard, and A. Vourlidas. Modeling of flux rope coronal mass ejections. Astrophys. J., 652, 763-773, 2006, DOI: 10.1086/508254.

Thompson, W.T., K. Wei, J.R. Burkepile, J.M. Davilla, and O.C. St. Cyr. Background subtraction for the SECCHI/COR1 telescope aboard STEREO. Sol. Phys., 262, 213-231, 2010, DOI: $10.1007 / \mathrm{s} 11207-010-9513-8$

Török, T., and B. Kliem. The evolution of twisting coronal magnetic flux ropes. Astron. Astrophys., 406, 1043-1059, 2003, DOI: 10.1051/0004-6361:20030692.

Török, T., and B. Kliem. Confined and ejective eruptions of kinkunstable flux ropes. Astrophys. J., 630, L97-L100, 2005, DOI: $10.1086 / 462412$.

Tousey, R. The solar corona. Space Research XIII, 2, 713-730, 1973.

Tripathi, D., V. Bothmer, and H. Cremades. The basic characteristics of EUV post-eruptive arcades and their role as tracers of coronal mass ejection source regions. Astron. Astrophys., 422, 337-349, 2004, DOI: 10.1051/0004-6361:20035815.

Webb, D.F. Erupting prominences and the geometry of coronal mass ejections. J. Geophys. Res., 93, 1749-1758, 1988, DOI: $10.1029 / \mathrm{JA} 093 \mathrm{iA03p01749.}$

Webb, D.F., and T.A. Howard. Coronal mass ejections: observations. Living Rev. Sol. Phys., 9, 3, 2012, DOI: $10.12942 /$ lrsp-2012-3.

Wood, B.E., and R.A. Howard. An empirical reconstruction of the 2008 April 26 coronal mass ejection. Astrophys. J., 702, 901-910, 2009, DOI: $10.1088 / 0004-637 X / 702 / 2 / 901$

Xie, H., L. Ofman, and G. Lawrence. Cone model for halo CMEs: application to space weather forecasting. J. Geophys. Res., 109, A03109, 2004, DOI: 10.1029/2003JA010226.

Yao, S., E. Marsch, C.-Y. Tu, and R. Schwenn. Identification of prominence ejecta by the proton distribution function and magnetic fine structure in interplanetary coronal mass ejections in the inner heliosphere. J. Geophys. Res., 115, A05103, 2010, DOI: $10.1029 / 2009 J A 014914$.

Zhang, J., and J. Wang. Filament eruptions and halo coronal mass ejections. Astrophys. J., 554, 474-487, 2001, DOI: $10.1086 / 321343$.

Zhang, M., and B.C. Low. Magnetic energy storage in the two hydromagnetic types of solar prominences. Astrophys. J., 600, 1043-1051, 2004, DOI: 10.1086/379891.

Cite this article as: Howard T. Regarding the detectability and measurement of coronal mass ejections. J. Space Weather Space Clim., $\mathbf{5}$, A22, 2015, DOI: $10.1051 /$ swsc/2015024. 